\title{
Le « consensus d'Antalya »: les avancées de la Plateforme intergouvernementale scientifique et politique sur la biodiversité et les services écosystémiques (IPBES)
}

\author{
Denis Pesche ${ }^{1}$, Mohamed Oubenal ${ }^{2}$, Jean-Christophe Vandevelde ${ }^{3}$, Marie Hrabanski ${ }^{4}$ \\ 1 Sociologue, CIRAD, UMR ART-Dev, 34090 Montpellier, France \\ 2 Sociologue, post-doctorant, CNRS, UMR CERIC, 13628 Aix-en-Provence, France \\ 3 Secrétaire scientifique du Comité national IPBES, FRB, 75005 Paris, France \\ 4 Sociologue, CIRAD, UMR ART-Dev, 34090 Montpellier, France
}

\section{Mots-clés :}

Biodiversité ; services écosystémiques ; IPBES

\section{Keywords:}

Biodiversity; ecosystem services; IPBES
Résumé - Après avoir retracé les grandes étapes qui ont conduit à la création de la Plateforme intergouvernementale sur la biodiversité et les services écosystémiques (IPBES), fonctionnelle depuis début 2013, cet article décrit les points-clés de la seconde session plénière d'IPBES qui s'est tenue à Antalya, en Turquie, en décembre 2013. Une série de décisions rangées sous l'appellation de « consensus d'Antalya » ont été prises par des représentants de 115 États dans trois grands domaines : le programme de travail pour les cinq années à venir (2014-2018), les règles et procédures de fonctionnement de la plateforme ainsi que les engagements financiers permettant de lui conférer l'autonomie suffisante pour son fonctionnement. D'autres questions sont restées en suspens, notamment celle de la place accordée aux acteurs non étatiques dans ce processus intergouvernemental. La troisième session plénière d'IPBES aura lieu à Bonn du 12 au 17 janvier 2015 : elle devrait, entre autres, débattre de la thématique de la pollinisation et de la production de nourriture.

\begin{abstract}
The "Antalya consensus": Advances of the Intergovernmental Platform on Biodiversity and Ecosystem Services (IPBES). After a brief history of the Intergovernmental Platform on Biodiversity and Ecosystem Services (IPBES), which is operational since early 2013, our paper describes the key points of the second plenary session of IPBES held in Antalya, Turkey, in December 2013. A raft of decisions known as "Antalya consensus" has been taken by representatives of 115 states in three main areas: the work program for the next five years (2014-2018), the rules and procedures for the future development of the platform and financial and in-kind support to manage this work program. Other issues remained unresolved, including that of the importance given to non-state actors in the intergovernmental process. The third plenary session of IPBES will take place in Bonn from 12 to 17 January 2015. Among other topics, the theme of pollinators, pollination and food production is to be addressed.
\end{abstract}

La seconde session plénière de la Plateforme intergouvernementale sur la biodiversité et les services écosystémiques (IPBES) s'est tenue à Antalya, en Turquie, du 9 au 14 décembre $2013^{1}$. Plus de 700 participants, représentant 115 États, étaient présents, ainsi que plusieurs dizaines d'observateurs. Une série de décisions rangées sous l'appellation de "consensus d'Antalya » ont été prises dans trois grands domaines: le programme de travail pour les cinq années à venir (20142018), les règles et procédures de fonctionnement de la plateforme et les engagements financiers permettant de lui conférer l'autonomie suffisante pour son fonctionnement. D'autres questions sont restées en suspens, notamment celle de la place accordée aux acteurs non étatiques

Auteur correspondant : D. Pesche, denis.pesche@cirad.fr

1 Ce texte s'inscrit dans le cadre de nos recherches menées au sein du projet Invaluable, financé par ERA-Net, et du projet Circulex, financé par l'ANR (voir encadré). 


\section{Encadré. IPBES : un terrain d'étude privilégié pour la recherche}

Notre participation à cette session plénière d'IPBES s'inscrit dans trois processus de recherche. Le plus directement concerné est le projet Circulex², financé par l'ANR (2013-2015) et qui rassemble des juristes et des chercheurs en sciences sociales autour de la question de la complexité institutionnelle de la gouvernance environnementale globale. La notion de régime international a été forgée pour saisir l'ensemble des accords et acteurs liés à la résolution d'un problème global. La multiplication des arènes internationales dans le domaine environnemental et leur enchevêtrement partiel a conduit à l'émergence récente de la notion de « complexe de régime ».

L'objet du projet Circulex est de saisir cette complexité par une entrée sur la circulation des normes et des acteurs entre différents régimes environnementaux, plus particulièrement celui de la biodiversité et celui du climat. Nous sommes ainsi engagés dans un travail de recherche visant à comprendre l'implication des acteurs non étatiques (souvent qualifiés de "stakeholders ») dans ces complexes de régimes. Version internationale du paradigme de la participation, cet appel à l'engagement des stakeholders dans la gouvernance environnementale globale ne va pas de soi. Ils ne forment pas un groupe homogène puisque des représentants d'ONG de conservation côtoient des représentants du secteur privé, des peuples indigènes ou encore des organisations scientifiques. Ils se caractérisent par des formes d'engagement et des manières d'agir très différentes, basées sur et produisant de fortes asymétries. Ces organisations et leurs représentants contribuent de fait à véhiculer, ou freiner, la circulation de normes, d'idées au sein des régimes mais aussi entre les régimes. Pour saisir la circulation et les asymétries entre les différents stakeholders mobilisés, nous avons profité de la session plénière d'Antalya pour réaliser des questionnaires d'analyse de réseaux sociaux (social network analysis) auprès de la population étudiée. IPBES constitue un terrain d'étude privilégié pour ces questions dans la mesure où, d'une manière plus prononcée que sur les questions climatiques, les stakeholders sont appelés à participer plus directement au processus lui-même.

D'autres collègues du projet Circulex analysent les relations entre les membres du bureau et du GEM d'IPBES pour comprendre les dynamiques qui s'y déploient, notamment en termes de confrontation entre les mondes du développement, de la conservation de la biodiversité, de l'économie...

Au-delà des problématiques abordées dans le projet Circulex sur le processus d'IPBES et ses stakeholders, une seconde question est explorée et concerne l'évolution des cadres d'analyse mobilisés pour saisir le lien entre la biodiversité et le bien-être humain. Le cadre proposé par le Millennium Ecosystem Assessment (2005) a mis en avant la notion de service écosystémique, laquelle a, quelques années plus tard, été directement intégrée dans le nom même de la plateforme intergouvernementale sur la biodiversité et les services écosystémiques (IPBES). Toutefois, le travail de confrontation et de construction de compromis sur les différentes visions de la nature se poursuit au sein d'IPBES et semble aboutir à une reconnaissance plurielle des rapports entre l'homme et la nature (suite du projet Serena ${ }^{3}$ financé par l'ANR, 2009-2013).

Enfin, une troisième question a trait aux critiques développées quant à l'usage des instruments de marché de la biodiversité (market based instruments [MBI]). Ce type d'instrument est à la fois souvent invoqué, mobilisé et critiqué dans les arènes de la biodiversité. Tandis que certains acteurs y sont farouchement opposés, d'autres sont plus nuancés et reconnaissent avant tout la diversité des instruments de marché de la biodiversité. La catégorie de MBI recouvre en effet une palette d'instruments assez diversifiée (taxes environnementales, mesures agrienvironnementales, paiements pour services environnementaux, compensation...). Nos recherches nous amènent à analyser plus en détail les différentes formes de la critique envers ces types d'instruments en déconstruisant la catégorie de MBI (projet Invaluable ${ }^{4}$ financé par ERA-Net, 2012-2014).

Denis Pesche et Marie Hrabanski

2 Circulations de normes et réseaux d'acteurs dans la gouvernance internationale de l'environnement : http:/ / circulex.wix.com/ projet.

3 Services environnementaux et usages de l'espace rural : http:/ / www.serena-anr.org.

4 http://invaluable.fr. 
dans ce processus intergouvernemental. Avant de revenir sur le contenu de cette rencontre, un rapide historique permet de la situer dans le processus plus large de la construction du régime international de la biodiversité.

\section{Bref historique du processus ayant amené à la création d'IPBES}

Depuis le début des années 1990, le domaine de l'environnement fait l'objet d'exercices d'évaluation environnementale globale ayant pour objectif de faire l'état des connaissances sur une question donnée afin de fournir aux décideurs des éléments pouvant servir à étayer leurs décisions ${ }^{5}$. Le plus connu de ce type d'exercice est celui réalisé par le GIEC ${ }^{6}$ qui, depuis 1990, publie régulièrement des rapports synthétisant les connaissances sur le changement climatique ${ }^{7}$.

Sur les questions de biodiversité, le Global Biodiversity Assessment (GBA) constitue le premier exercice d'évaluation scientifique d'envergure, réalisé entre 1993 et $1995^{8}$. Il n'aura toutefois pratiquement pas d'impact sur la formulation des politiques car il est conduit comme un exercice non gouvernemental, sans appropriation par les pouvoirs publics ${ }^{9}$. Quelques années plus tard, le Millennium Ecosystem Assessment (MEA) va au contraire veiller à inclure les décideurs dans le processus d'évaluation. Publiés en 2005, les résultats du MEA vont avoir un large écho dans les communautés scientifiques et politiques de la biodiversité ${ }^{10}$. Dès lors, les promoteurs du MEA s'investissent massivement en faveur de la diffusion de leurs travaux et s'engagent dans la promotion

5 Mitchell, R.B., Clark, W.C., Cash, D.W., Dickson, N.M. (Eds), 2006. Global environmental assessments: information and influence, Cambridge [MA], MIT Press.

6 Le GIEC (Groupe d'experts intergouvernemental sur l'évolution du climat) a été créé en 1988 et cinq rapports sur le changement climatique ont été publiés depuis (1990, 1995, 2001, 2007 et 2014).

7 Dahan, A., 2009. Entre Poznan et Copenhague : le régime climatique au milieu du gué, Natures Sciences Sociétés, 17, 3, 271-282.

8 Voir Heywood, V.H. (Ed.), 1996. Global Biodiversity Assessment, New York, Cambridge University Press.

9 Watson, R.T., 2005. Turning science into policy: challenges and experiences from the science-policy interface, Phil. Trans. R. Soc. $B, 360,1454,471-477$.

10 Pesche, D., 2013. Le Millennium Ecosystem Assessment : anatomie d'une évaluation environnementale globale, Natures Sciences Sociétés, 21, 4, 363-372 ; Hrabanski, M., 2013. Les experts scientifiques français dans le Millenium Ecosystem Assessment (2001-2005) : les raisons de leur absence, Natures Sciences Sociétés, 21, 2, 182-189. de l'idée d'un mécanisme d'évaluation scientifique des écosystèmes. Ainsi, dès 2005, Diversitas ${ }^{11}$, qui se présente comme le porte-parole de la communauté scientifique sur les questions de biodiversité, appelle à la mise en place «d'un mécanisme international d'expertise sur la biodiversité dans la lignée du groupe existant sur le changement climatique (Groupe d'experts intergouvernemental sur l'évolution du climat [GIEC $\left.{ }^{12}\right]$ ) ». Parallèlement, la même année, la France prend l'initiative d'organiser avec le soutien de l'Unesco une conférence internationale, "Biodiversité, science et gouvernance », qui donnera l'impulsion d'un processus de consultation internationale multi-acteurs (IMoSEB ${ }^{13}$ ) pour évaluer les besoins en connaissances scientifiques et les options politiques dans le domaine de la biodiversité. L'émergence d'IPBES résulte de la convergence de ces deux processus : l'initiative IMoSEB (2006-2007) et les suites du $\mathrm{MEA}^{14}$. En 2008, la Conférence des parties de la Convention sur la diversité biologique (CDB) prend l'option d'amorcer un processus de construction d'une plateforme multi-acteurs pour améliorer l'interface sciencepolitique sur la biodiversité. Cette initiative est placée sous la coordination du Programme des Nations unies pour l'environnement (PNUE) et aboutira à la rédaction $\mathrm{d}^{\prime}$ une feuille de route pour IPBES ${ }^{15}$. Ensuite, deux sessions préparatoires (Nairobi, 2011 et Panama, 2012) jetteront les bases $\mathrm{du}$ processus plus formel, associant 94 pays. L'option est prise d'implanter le secrétariat $\mathrm{d}^{\prime} \mathrm{IPBES}$ à Bonn et $\mathrm{d}^{\prime} \mathrm{y}$ tenir la première session plénière en janvier 2013. Cette première plénière est l'occasion de mettre en place les instances de gouvernance d'IPBES : le

\footnotetext{
${ }^{11}$ Diversitas est un réseau international de recherche lancé en 1992 par l'ICSU (International Council for Science) en collaboration avec l'Unesco et l'Union internationale des sciences biologiques (UISB ; en anglais, International Union of Biological Sciences, IUBS).

12 Locatelli, B., Babin, D., Torquebiau, E., 2009. Colloques et documents, comptes rendus : "Biodiversity and society: understanding connections, adapting to change" (Colloque, Le Cap, Afrique du Sud, 13-16 octobre 2009), Natures Sciences Sociétés, 18, 4, 462-465.

13 IMoSEB: International Mechanism of Scientific Expertise on Biodiversity.

${ }^{14}$ Larigauderie, A., Mooney, H., 2010. The Intergovernmental science-policy Platform on Biodiversity and Ecosystem Services: moving a step closer to an IPCC-like mechanism for biodiversity, Current Opinion in Environmental Sustainability, 2, 1-6.

15 Cette feuille de route est souvent appelée «Busan Outcomes » évoquant la ville coréenne où s'est tenue la réunion qui en a défini le contenu (Duraiappah, A.K., Rogers, D., 2011. The Intergovernmental Platform on Biodiversity and Ecosystem Services: opportunities for the social sciences, Innovation: The European Journal of Social Science Research, 24, 3, 217-224; Goerg, C., Nesshöver, C., Paulsch, A., 2010. A new link between biodiversity science and policy, GAIA, 19, 3, 183-186).
} 
malais Abdul Hamid Zakri préside la plateforme et IPBES se dote d'un bureau, d'un groupe d'experts multidisciplinaire (GEM) et d'un secrétariat intérimaire.

\section{Débats et résultats de la seconde plénière}

La plénière d'Antalya a traité principalement (1) du programme de travail 2014-2018 et du cadre conceptuel d'IPBES, (2) des arrangements institutionnels pour le fonctionnement de la plateforme, et (3) du budget nécessaire pour permettre ce fonctionnement. Pendant ces 6 jours de travail, les séances plénières ont alterné avec des sessions de travail en trois "groupes de contact» parallèles : le premier portait sur le programme de travail et le cadre conceptuel, le deuxième sur les procédures de fonctionnement et le troisième sur le budget. Chaque matin, les pays se réunissaient également par grandes régions afin de se concerter avant le démarrage des travaux en séance plénière et en groupes de travail ${ }^{16}$.

\section{Le programme de travail et le cadre conceptuel d'IPBES}

Le programme de travail d'IPBES pour les cinq prochaines années (2014-2018) se propose de répondre à quatre grandes fonctions: (a) identifier et hiérarchiser les principales informations scientifiques dont les décideurs ont besoin et promouvoir la production de nouvelles connaissances sans entreprendre directement de nouvelles recherches, (b) conduire des évaluations périodiques de l'état des connaissances en matière de biodiversité et de services écosystémiques et sur des thèmes spécifiques aux niveaux mondial, régional et sous-régional, (c) appuyer l'élaboration et l'exécution des politiques en identifiant des outils et des méthodes appropriés et (d) hiérarchiser les besoins en matière de création de capacités et fournir des appuis en vue d'améliorer l'interface science-politique.

Pour mener à bien ces quatre fonctions, quatre objectifs ont été identifiés.

- Renforcer les capacités et les connaissances à l'interface science-politique pour que la plateforme puisse $\mathbf{s}^{\prime}$ acquitter de ses principales fonctions ${ }^{17}$ (objectif 1 ).

\footnotetext{
${ }^{16}$ Les participants ont débattu sur la base de 27 documents de travail et 19 documents d'information, tous téléchargeables sur le site d'IPBES : http://ipbes.net/plenary/ipbes-2.html. Des comptes rendus détaillés de cette seconde réunion plénière d'IPBES sont consultables sur le site de l'Institut international $\mathrm{du}$ développement durable (IISD) : http://www.iisd.ca/ ipbes/ipbes2/.

17 Cet objectif transversal mais aussi les deux suivants sont explicitement reliés à plusieurs « objectifs d'Aichi » élaborés en 2010 dans le cadre de la CDB.
}

Par rapport au GIEC, cet objectif transversal est une spécificité $\mathrm{d}$ 'IPBES répondant à la demande de pays en développement. La réunion d'Antalya a ainsi acté la mise en place de trois équipes spéciales (task force). La première travaillera sur le développement des compétences (capacity building) et aura pour objectif de mettre en place des programmes de formation et des bourses, surtout pour les experts des pays en voie de développement afin que ceux-ci puissent participer aux évaluations de la plateforme. Une seconde équipe spéciale s'intéressera aux systèmes de connaissances locaux et autochtones en vue de leur intégration dans les évaluations futures. La troisième aura pour tâche d'identifier les besoins prioritaires en termes d'outils de production et de gestion des connaissances et des données sur la biodiversité.

- Renforcer l'interface science-politique aux niveaux régional et global (objectif 2). Ce chantier va démarrer par un guide sur l'intégration des évaluations à différentes échelles (fin 2014) puis des évaluations sousrégionales et régionales seront lancées (en 2015), qui mèneront à une évaluation globale, prévue pour décembre 2018.

- Renforcer l'interface science-politique sur des questions thématiques (objectif 3). Plusieurs évaluations thématiques vont être lancées en 2014 : la première est une évaluation accélérée sur « la pollinisation et les pollinisateurs associés à la production alimentaire ». Trois autres évaluations concernent « la dégradation des terres et leur restauration », " les espèces exotiques envahissantes » et "l'utilisation durable de la biodiversité ». Deux évaluations à caractère méthodologique sont également prévues au programme: l'une sur les outils et méthodes d'appui à la décision par l'analyse des scénarios et la modélisation de la biodiversité et des services écosystémiques ; l'autre sur les outils et méthodes d'évaluation de la biodiversité, à partir de différentes conceptualisations des valeurs de la biodiversité et des bénéfices apportés par la nature.

- Faire connaître et évaluer IPBES, ses produits et ses conclusions (objectif 4). Cela comprend en particulier l'élaboration de répertoires des évaluations et outils d'aide à la décision existants mais aussi des outils et méthodes d'appui aux politiques. La politique de communication et l'évaluation à mi-parcours des activités entreprises sont incluses dans cet objectif.

Les délégués ont aussi discuté la proposition de cadre conceptuel, qui doit guider les travaux d'IPBES. Plusieurs réunions courant 2012 avaient permis d'en élaborer une première version volontairement simplifiée en vue des premières discussions d'IPBES à Bonn en janvier 2013. Ce cadre a ensuite beaucoup évolué suite à un travail de consultation et de synthèse : les délégations présentes à Antalya ont pu se mettre d'accord sur une version améliorée qui leur avait été soumise en préparation de cette seconde réunion plénière, et qui 
intègre différentes conceptions de la nature et des liens avec les sociétés, notamment celle liant écosystèmes et services écosystémiques ainsi que l'approche de la «Terre Mère » et des dons de la nature défendue notamment par la Bolivie.

\section{Mécanismes institutionnels et gouvernance d'IPBES}

La mise en œuvre opérationnelle de ces quatre objectifs repose sur les instances organisationnelles formelles d'IPBES. La plénière, organe politique principal, assume la responsabilité globale du programme de travail et validera les produits à venir. Elle a également mandaté un bureau de 16 personnes pour piloter le programme d'activités, préparer et animer les séances plénières. Ce bureau, organe politique délégué par la plénière, assure la coordination et le cadrage général. Un groupe d'experts multidisciplinaire (GEM), composé de 25 scientifiques issus des principales régions du monde, constitue un organe à caractère consultatif mais qui joue un rôle-clé sur les questions scientifiques (choix des experts, par exemple). Enfin, le secrétariat assure le bon fonctionnement de la plateforme et appuie les instances d'IPBES dans leurs fonctions. La plénière d'Antalya a d'ailleurs été l'occasion de la nomination de la Française Anne Larigauderie (Diversitas) à la tête du nouveau secrétariat.

L'une des questions âprement débattues à Antalya a été le processus de nomination des membres du GEM ${ }^{18}$. Plusieurs États ont vivement critiqué une proposition qui laissait une marge de manœuvre au bureau dans leur sélection, cela dans le but d'obtenir un équilibre (entre disciplines, hommes-femmes). Cette initiative contestée a surtout fait apparaître, dès le début de la plénière, la grande méfiance de plusieurs délégations à l'égard du bureau d'IPBES, notamment en raison du risque qu'il puisse influer sur les travaux du GEM, mettant en péril son «indépendance scientifique». Les États membres ont donc largement opté pour l'autre proposition selon laquelle chacune des cinq régions des Nations unies désigne 5 candidats pour le GEM. Au travers de consultations au sein de chaque région, puis entre régions, un équilibre dans la représentation des sexes et des différentes disciplines sera néanmoins encouragé.

Une autre question très débattue a été la place des experts nominés par des acteurs non étatiques (ONG, scientifiques, secteur privé, représentants des peuples autochtones...). Des observateurs tels que $1^{\prime}$ UICN (Union internationale pour la conservation de la nature), Diversitas et la Society for Conservation Biology (SCB), soutenus par des pays développés, voulaient donner le

\footnotetext{
${ }^{18}$ Le GEM a été mis en place après la première plénière de Bonn en janvier 2013 pour un mandat de deux ans.
}

droit aux différentes parties prenantes (stakeholders ${ }^{19}$ ) de participer à la nomination aussi bien des membres du GEM que des experts sollicités pour les différentes évaluations. Néanmoins, plusieurs pays émergents et en voie de développement s'y sont opposés. Certains pays comme l'Argentine, la Chine ou la Russie, ont considéré cela comme une ingérence dans des questions qui relèvent, pour eux, de la souveraineté nationale. Après plusieurs rounds de négociations, un compromis a été obtenu. Celui-ci confère aux seuls États la possibilité de nominer des membres pour le GEM. Concernant les experts à nominer pour les différentes évaluations, jusqu'à $20 \%$ d'entre eux pourront provenir des parties prenantes qualifiées (relevant stakeholders). Certains observateurs craignent toutefois que la mainmise des États sur la nomination des membres du GEM conduise à reproduire le schéma du SBSTTA, organe scientifique de la Convention sur la diversité biologique, qui fait l'objet de nombreuses critiques liées aux tensions politiques qui prévalent souvent sur son objectif premier consistant à apporter un conseil scientifique et une expertise sur les questions de biodiversité.

Par ailleurs, les acteurs non étatiques ont été déçus par l'ajournement de la discussion autour d'une stratégie de leur mobilisation et d'une stratégie de communication. Alors même qu'il leur est demandé d'apporter des contributions en nature et en connaissances pour la réalisation du programme de travail de la plateforme, ils regrettent que leur pouvoir relatif à la nomination d'experts soit réduit et qu'aucune structure ne soit créée pour les associer plus formellement.

Pour autant, la place des acteurs non étatiques est abordée de façon assez originale dans IPBES, même si elle fait débat. Depuis les premières réunions d'IPBES, en 2008, ils ont participé au processus aux côtés des représentants des États, des organisations onusiennes et des conventions internationales concernées. Après la première plénière de Bonn, en janvier 2013, l'option a été prise de les impliquer d'une manière plus effective. Le PNUE, qui assurait le secrétariat intérimaire d'IPBES, a mandaté l'ICSU et l'UICN pour préparer et animer une réunion des parties prenantes qui s'est tenue juste avant la seconde réunion plénière, les 7 et 8 décembre 2013 . Plusieurs réunions régionales avaient également été organisées au préalable dans les 5 régions onusiennes. Le secteur privé semble très peu présent à ce stade. Ce sont principalement des organisations scientifiques et liées à la conservation qui sont actives (l'ICSU et l'UICN en tête, mais aussi la SCB) avec une participation remarquée des

\footnotetext{
${ }^{19}$ Dans ce texte, nous utilisons aussi la notion d'acteurs non étatiques pour qualifier ces parties prenantes bien que, pour IPBES, les stakeholders puissent aussi compter des instances publiques comme des musées, par exemple, ou des instituts de recherche.
} 
organisations représentant les peuples indigènes et les communautés locales, qui ne souhaitent pas être considérées comme « parties prenantes » mais revendiquent le statut spécifique de partenaires. Du côté des États, la plupart des pays développés estiment qu'IPBES doit être une instance inclusive intégrant des organisations scientifiques et des ONG reconnues, l'enjeu étant l'indépendance et l'excellence scientifique, garantes de la qualité des travaux. En revanche, pour plusieurs pays du G77, les règles onusiennes doivent s'appliquer strictement, seuls les États membres étant légitimes pour gouverner le processus.

Pour compléter ce dispositif, un arrangement institutionnel a été mis en place entre la plénière et les quatre organisations des Nations unies concernées par les activités d'IPBES (PNUE, Unesco, FAO et PNUD). Cet arrangement formalise un lien fort entre la plateforme et le système des Nations unies, ce qui était attendu notamment par les pays africains. De plus, il permet à des acteurs comme l'Unesco et la FAO de se sentir moins marginalisés par rapport au PNUE, qui joue depuis le début un rôle moteur dans IPBES.

\section{Budget et questions financières}

Enfin, sur le plan budgétaire, la plénière a adopté un budget pour 2014-2015 et un budget prévisionnel indicatif pour 2016-2018. Les dépenses prévues s'élèvent à environ 7 millions de dollars américains pour 2014 et 8 millions pour 2015. Ces dépenses, contrairement au sentiment largement partagé en début de plénière, vont pouvoir être couvertes du fait des contributions de plusieurs pays membres, dont principalement la Norvège (8 M\$ payés en 2013) mais aussi l’Allemagne (1 300000 \$ chaque année jusqu'en 2018), la Grande-Bretagne (640 000 \$ pour 2014), les États-Unis (500 $000 \$$ pour 2014), les Pays-Bas (690 $000 \$)$ et la France (275 $000 \$)$, ainsi que du report important des contributions versées au titre de 2012 et 2013 (13,5 M\$). Il faut cependant noter que seulement 10 pays ont été en mesure d'annoncer des contributions lors de cette seconde réunion plénière.

\section{Principaux questionnements}

L'un des principaux enjeux d'IPBES est l'articulation des questions de conservation et de développement ${ }^{20}$. Des représentants du G77 ont d'ailleurs rappelé, lors de discussions en marge de l'événement, que, dans leur pays, ils peinent à convaincre de la pertinence de la conservation de la biodiversité, celle-ci étant souvent plutôt perçue comme un frein au développement économique. Or, le lien entre la protection de la biodiversité et

\footnotetext{
${ }^{20}$ Duraiappah, A.K., Rogers, D., 2011, op. cit.
}

les questions de développement n'a pas été réellement débattu ni envisagé lors de la plénière d'Antalya. Chez les acteurs non étatiques, aucune association de développement n'a fait le déplacement et le PNUD n'est intervenu sur le sujet qu'au travers d'un événement «off » coorganisé avec la Norvège.

Les participants et les observateurs d'IPBES rappellent souvent l'analogie qui existe avec le GIEC. Sa renommée permet ainsi de concevoir une structure et un mode de fonctionnement basés sur une expérience jugée réussie. Mais la construction d'IPBES ne se fait pas vraiment à l'identique du GIEC et comporte des spécificités qui font sa singularité. Les communautés locales et indigènes, de par leurs connaissances en matière de gestion et de conservation de la biodiversité, occupent une place stratégique dans la CDB. Par conséquent, la nouvelle plateforme accorde une importance à l'intégration des savoirs provenant de ces groupes.

En outre, à la différence des évaluations sur le changement climatique, l'érosion de la biodiversité s'observe d'abord à une échelle locale, mais aussi à des échelles plus larges, ce qui plaide en faveur de la mise en place de programmes de travail régionaux et nationaux, lesquels devront être coordonnés via IPBES à travers des modalités permettant l'articulation de ces différentes échelles.

Enfin, la vision exclusivement économique et utilitariste de la nature est discutée dans le processus d'IPBES. En effet, le cadre conceptuel intègre les différences de représentations de la nature et prend en compte à la fois l'approche en termes de services écosystémiques et les dimensions culturelles et sacrées qui peuvent lui être attribuées. Cette vision plurielle de la nature plaide en faveur d'une ouverture disciplinaire d'IPBES, qui ne soit pas seulement dominée par les sciences de la nature mais qui s'ouvre également aux recherches en sciences sociales, ainsi qu'aux détenteurs de savoirs locaux et autochtones.

\section{La place de la France dans ce processus}

Bien que sur le plan financier, la France ne fasse pas partie des pays de premier plan, elle est l'un des États les plus influents sur les positions prises par l'Union européenne, notamment concernant l'implication plus grande des parties prenantes non étatiques. Elle a aussi réussi, par exemple, à intégrer au cadre conceptuel d'IPBES la notion de fonctionnement des écosystèmes qui vise à préciser les liens entre biodiversité et services écosystémiques. Elle arrive également à construire des positions communes avec quelques pays sur des questions précises, comme l'importance de l'évaluation de la biodiversité marine. Si la France a une tradition scientifique importante sur les questions de biodiversité et une 
capacité à agir internationalement (comme elle l'a démontré en facilitant la création d'IMoSEB, et donc d'IPBES), elle s'est par contre très peu impliquée dans le MEA et reste, pour l'essentiel de ses communautés scientifiques, encore peu mobilisée sur les analyses privilégiant une approche en termes de services écosystémiques. Cependant, au niveau national, un important travail d'évaluation des écosystèmes et des services écosystémiques (EFESE) vient d'être lancé par le ministère de l'Écologie, du Développement durable et de l'Énergie (MEDDE) et s'inscrit dans le cadre de la Stratégie européenne de la biodiversité. De même, un comité national
IPBES, composé de représentants des ministères concernés, des organismes de recherche et des porteurs d'enjeux sur les questions de biodiversité, a été créé en 2013 dans l'objectif de mobiliser la communauté des scientifiques et des experts français en vue des travaux de la plateforme. L'enjeu maintenant est de relier ces projets développés à des échelles diverses, et de convaincre les communautés scientifiques françaises de s'impliquer dans des exercices multidisciplinaires, voire multi-acteurs, exercices qui ne sont pas forcément toujours reconnus dans la gestion des carrières des chercheurs. Vaste défi ! 